\section{O Sistema de Vigilância Alimentar e Nutricional como instrumento de monitoramento da Estratégia Nacional para Alimentação Complementar Saudável}

\section{The Food and Nutrition Surveillance System as a tool for monitoring the National Strategy for Healthy Complementary Nutrition}

Carolina Souza Ferreira 1

Mariangela Leal Cherchiglia 2

Cibele Comini César 3

\section{Abstract}

Objectives:to examine the Food and Nutrition Surveillance System (Sisvan) as a tool for monitoring the National Strategy for Healthy Complementary Nutrition (ENPACS) in the 40 municipalities overseen by the Belo Horizonte regional superintendent for health (SRS-BH).

Methods: a descriptive study was carried out involving all children aged under two being accompanied by the Sisvan Web between 2008 and 2011. The coverage of the Sisvan Web was calculated by dividing the number of children aged under two years accompanied by the Sisvan Web by the total population for the same age group. A questionnaire relating to the technical references of the Sisvan of the municipalities under investigation was sent to collect information on the functioning of the Sisvan.

Results: the coverage of the Sisvan Web, in all municipalities, varied from $4.3 \%$ (2008) to $10.7 \%$ (2011). The questionnaire was answered by 38 municipalities in the SRS-BH, 31.6\% of whom reported using data from the Sisvan Web system as a basis for nutritional interventions.

Conclusions: the study identified low coverage, poor utilization of data and the need to improve the Sisvan, in order to generate consistent information on nutrition and food among children aged under two years, thereby making it appropriate for monitoring of the ENPACS.

Key words Nutrition assessment, Food consumption, Infant, Information systems, Nutritional surveillance
1-3 Universidade Federal de Minas Gerais. Av. Antônio Carlos, 6627. Pampulha. Belo Horizonte, MG, Brasil. CEP: 31.270-901.

E-mail: carolinanutry@yahoo.com.br
Resumo

Objetivos: analisar o Sistema de Vigilância Alimentar e Nutricional (Sisvan) como instrumento de monitoramento da Estratégia Nacional para Alimentação Complementar Saudável (ENPACS) nos 40 Municipios da Superintendência Regional de Saúde de Belo Horizonte (SRS-BH).

Métodos: realizou-se estudo descritivo envolvendo o total de crianças menores de dois anos acompanhadas pelo Sisvan Web nos anos de 2008 a 2011. A cobertura do Sisvan Web foi calculada dividindo-se o número de crianças menores de dois anos, acompanhadas pelo Sisvan Web, pela população na mesma faixa etária. Foi enviado questionário pertinente às referências técnicas do Sisvan dos municípios avaliados, para levantamento de informações sobre o funcionamento do Sisvan.

Resultados: a cobertura do Sisvan Web, na totalidade dos municípios, variou de 4,3\% (2008) a 10,7\% (2011). O questionário foi respondido por 38 Municípios da SRS-BH, sendo que desses, 31,6\% informaram utilizar os dados do Sisvan Web para estabelecer ações de intervenção nutricional.

Conclusões: o estudo identificou baixas coberturas, pouca utilização dos dados e a necessidade de fortalecer o Sisvan, para que possa gerar informações consistentes sobre a situação alimentar $e$ nutricional das crianças menores de dois anos, tornando-se, assim, adequado para o monitoramento da ENPACS

Palavras-chave Avaliação nutricional, Consumo de alimentos, Lactente, Sistemas de informação, vigilância nutricional 


\section{Introdução}

Os Sistemas de Informação em Saúde (SIS) são mecanismos para a obtenção de informação mediante coleta, registro, processamento e análise de dados; transformação do dado em informação e oportuna divulgação. Os SIS têm como objetivo facilitar a formulação e avaliação das políticas, planos e programas de saúde, fornecendo subsídios ao processo de tomada de decisões. ${ }^{1}$ A informação orienta a implantação, o acompanhamento e a avaliação das ações de prevenção e controle de doenças, sendo a base para a gestão dos serviços de saúde. Por isso, é fundamental que as informações em saúde tenham qualidade e retratem de forma fidedigna a situação de saúde nos diversos Estados e Municípios brasileiros. ${ }^{2}$

Instrumento de apoio às ações de promoção da saúde, o Sistema de Vigilância Alimentar e Nutricional (Sisvan) é oferecido aos profissionais de saúde e aos gestores do Sistema Único de Saúde (SUS), visando à melhoria da qualidade da assistência à população. ${ }^{3} \mathrm{O}$ Sisvan é um SIS que tem como objetivo a geração de informações sobre a situação alimentar e nutricional da população, contribuindo para o conhecimento da natureza e da magnitude dos problemas de nutrição, identificando áreas geográficas, segmentos sociais e grupos populacionais de maior risco aos agravos nutricionais. ${ }^{4}$ Essas informações deverão fornecer subsídios aos responsáveis por políticas que visam à melhoria dos padrões de consumo alimentar e do estado nutricional da população. Dessa forma, o Sisvan auxiliará os gestores públicos na formulação e monitoramento de políticas de alimentação e nutrição. 5

O Sisvan foi estabelecido nacionalmente no ano 1990, após a promulgação da Lei 8080/1990 e a publicação da Portaria n ${ }^{\circ} 1156$ de 31 de agosto do mesmo ano. No ano de 2002, iniciou-se o processo de informatização do Sisvan no Brasil, que culminou com a criação do Sisvan Módulo Municipal em 2003.6 Estudos têm apontado limitações do Sisvan, tais como: baixa cobertura geográfica e populacional 7,8 ; descontinuidade no envio de $\operatorname{dados}^{9,10}$; sustentação política pouco consistente 7,11 ; e dados produzidos que não acionam intervenções dos governos. 7,12 Em 2008, o Sisvan Web, que tem como objetivo o acompanhamento do estado nutricional e do consumo alimentar dos indivíduos atendidos pela Atenção Básica à Saúde, substituiu o Sisvan Módulo Municipal.13

Um dos segmentos populacionais foco do Sisvan são os lactentes. ${ }^{4} \mathrm{O}$ consumo alimentar na infância está associado ao perfil de saúde e nutrição. A prática alimentar inadequada, nos dois primeiros anos de vida, está associada ao aumento de morbidades como doenças infecciosas, desnutrição, excesso de peso e carências específicas de micronutrientes, tais como ferro, zinco e vitamina A.14 Resultados da II Pesquisa de Prevalência de Aleitamento Materno15 indicaram que o Brasil está distante do cumprimento das metas de aleitamento materno exclusivo até o sexto mês de vida e manutenção da amamentação, até o segundo ano de vida ou mais, propostas pela Organização Mundial da Saúde (OMS) e Ministério da Saúde (MS). Por meio dessa pesquisa também se verificou a necessidade de intervenções para a promoção de hábitos alimentares saudáveis no primeiro ano de vida.

Diante desse contexto, o MS, em parceria com a Rede Internacional em Defesa do Direito de Amamentar (Ibfan Brasil) e a Organização PanAmericana da Saúde (OPAS), elaborou a Estratégia Nacional para Alimentação Complementar Saudável (ENPACS). Trata-se de uma estratégia desenvolvida para fortalecer as ações de promoção à alimentação complementar saudável no SUS. A ENPACS tem como objetivo incentivar os profissionais de saúde da Atenção Básica $(\mathrm{AB})$ a realizarem a orientação alimentar para crianças menores de dois anos como atividade de rotina. 14

No ano de 2009, iniciou-se a realização de oficinas da ENPACS, dirigidas aos profissionais de saúde do SUS, para a formação de tutores da Estratégia.16 O tutor tem a missão de replicar o conteúdo da ENPACS, por meio da realização de novas oficinas para formação de tutores ou de oficinas, chamadas "rodas de conversa", para sensibilização sobre o tema nas Unidades Básicas de Saúde (UBS). É preconizado pelo MS que o monitoramento da ENPACS seja feito por meio do acompanhamento da replicação das oficinas e dos resultados alcançados pelos indicadores do Sisvan para crianças menores de dois anos. ${ }^{14,17}$

Considerando a importância e as limitações do Sisvan para o monitoramento das políticas de alimentação e nutrição do SUS e, que sua adequada implementação beneficiará gestores públicos, profissionais de saúde e a população, o presente estudo teve como objetivo analisar o Sisvan como instrumento de monitoramento da ENPACS e da situação alimentar e nutricional das crianças menores de dois anos que frequentam as UBS do SUS, nos Municípios da Superintendência Regional de Saúde de Belo Horizonte (SRS-BH). 


\section{Métodos}

Tratou-se de estudo descritivo que envolveu o total de crianças de 0 a 23 meses, público-alvo da ENPACS, acompanhadas pelo Sisvan $W e b$, no período de janeiro de 2008 a dezembro de 2011, nos Municípios da SRS-BH. O total de crianças acompanhadas pelos Sisvan $W e b$, em cada ano, foi: 4205 em 2008, 7281 em 2009, 8326 em 2010 e 8027 em 2011.

A SRS-BH é uma das 28 Regionais de Saúde da Secretaria de Estado de Saúde de Minas Gerais (SES-MG) e abrange $25,8 \% 18$ da população de Minas Gerais. Atualmente há 40 municípios sob jurisdição dessa regional: Belo Horizonte, Belo Vale, Betim, Bonfim, Brumadinho, Caeté, Conceição do Mato Dentro, Confins, Contagem, Crucilândia, Esmeraldas, Florestal, Ibirité, Igarapé, Itabirito, Jaboticatubas, Juatuba, Lagoa Santa, Mariana, Mário Campos, Mateus Leme, Matozinhos, Moeda, Nova Lima, Nova União, Ouro Preto, Pedro Leopoldo, Piedade dos Gerais, Raposos, Ribeirão das Neves, Rio Acima, Rio Manso, Sabará, Santa Luzia Santana do Riacho, São Joaquim de Bicas, São José da Lapa, Sarzedo, Taquaraçu de Minas e Vespasiano.

Durante agosto de 2012, foram coletados dados do Sisvan Web e do Sistema de Informação da Atenção Básica (SIAB). Os dados coletados foram: número de crianças de 0 a 23 meses acompanhadas, mensalmente e anualmente, pelo índice antropométrico peso/idade do Sisvan Web19; número de crianças avaliadas, anualmente, para construção do marcador de consumo alimentar do Sisvan Web ${ }^{19}$ "proporção de crianças de 0 a 6 meses sob aleitamento materno" e número de crianças de 0 a 23 meses pesadas, mensalmente, pelas equipes da $\mathrm{AB}$, de acordo com o Sistema de Informação da Atenção Básica. ${ }^{20}$

Os dados de estado nutricional das crianças menores de dois anos beneficiárias do Bolsa Família que são inseridos no Sistema de Gestão do Programa Bolsa Família (SGPBF) migram, semestralmente, para o Sisvan Web.21 Verificou-se o percentual de municípios que apresentaram acompanhamentos realizados para o índice peso/idade do Sisvan $\mathrm{Web}$, nos anos de 2008 a 2011, inseridos diretamente no Sisvan $W e b$ ou provenientes do SGPBF. No período analisado, apenas um município não apresentou dados no Sisvan $W e b$ e foi excluído das análises do tópico "Sisvan Web" e das análises de associação. Esse município possui sistema próprio de vigilância alimentar e nutricional e, apesar de alimentar o SGPBF, os dados não migram para o Sisvan $W e b$.

Como indicador do processo de alimentação do
Sisvan Web, verificou-se o número de crianças acompanhadas por mês para o índice peso/idade por sistema de origem do registro (Sisvan $\mathrm{Web}$ ou SGPBF). O ano 2008 foi excluído dessa análise, pois, durante o primeiro semestre desse ano houve a transição entre o Sisvan Módulo Municipal e o Sisvan Web.

A cobertura do Sisvan $W e b$ para crianças de 0 a 23 meses, em cada município, foi calculada por meio da proporção entre o número de crianças de 0 a 23 meses acompanhadas pelo índice peso/idade no ano e a população de crianças de 0 a 23 meses no mesmo ano $\mathrm{x} 100$. O numerador foi obtido diretamente dos relatórios do Sisvan Web.19 O denominador foi obtido por meio de acesso ao Tabnet do Departamento de Informática do SUS (Censo 2010 e projeções populacionais para os anos de 2008, 2009 e 2011). 18

A cobertura foi calculada para a regional como um todo e estratificando de acordo com categorias definidas pela população do município, segundo o Censo 2010. Foram definidos quatro grupos de municípios, com base na classificação de porte populacional do Instituto Brasileiro de Geografia e Estatística (IBGE)22: muito pequeno (população até 20.000 habitantes), pequeno (população de 20.001 a 50.000 habitantes), médio (população de 50.001 a 100.000 habitantes) e grande (população com mais de 100.000 habitantes)

Para a regional como um todo, a cobertura do Sisvan Web também foi calculada excluindo-se o número de beneficiários de planos de saúde 23 (dados do mês de junho dos anos de 2008, 2009, 2010 e 2011) do denominador. Utilizou-se o percentual de crianças de 0 a 11 meses beneficiárias de planos de saúde para estimar o número de crianças de 0 a 23 meses beneficiárias, pois este último dado não se encontra disponível no Tabnet da Agência Nacional de Saúde Suplementar (ANS).

Para verificar a cobertura do Sisvan, em relação às pesagens realizadas pelas equipes $\mathrm{da} A \mathrm{~B}$, foram calculadas as razões entre o total de crianças da SRS-BH acompanhadas, mensalmente, pelo índice peso/idade do Sisvan Web e o total de crianças da SRS-BH pesadas no mesmo período de acordo com o SIAB x 100. Nessa análise foram considerados os dados de 38 municípios da SRS-BH, pois também foi excluído um município que não possui equipe de $\mathrm{AB}$ e, por isso, não alimenta o SIAB.

Foi enviado "Questionário sobre o funcionamento do Sisvan" pertinente às referências técnicas do Sisvan dos municípios avaliados, com o objetivo de obter informações sobre a coleta, digitação, análise e utilização dos dados da vigilância alimentar 
e nutricional. Além disso, foram incluídas algumas perguntas sobre a replicação da ENPACS. Os dados dos questionários foram coletados em setembro e outubro de 2012.

Os dados referentes à replicação de oficinas da ENPACS nos municípios foram obtidos por meio de consulta às "Planilhas de monitoramento da ENPACS", enviadas pelos tutores da ENPACS à SRS-BH. Foram consideradas as informações das planilhas enviadas até agosto 2012.

A variável "replicação da ENPACS" foi analisada de acordo com as seguintes variáveis qualitativas: "analisar dados do Sisvan $\mathrm{Web}$ referentes a estado nutricional" e "estabelecer ações de intervenção com base nas análises dos dados do Sisvan Web". Nas análises que continham a variável "replicação da ENPACS" foram considerados apenas os municípios que tiveram tutor da ENPACS formado. Apenas os municípios que enviaram a planilha de monitoramento foram considerados replicadores da ENPACS

Os dados foram transferidos e analisados no software R. A tendência de aumento da cobertura no tempo foi avaliada por meio da análise do coeficiente de variação da equação de regressão quantílica da cobertura, com o ano e porte, avaliado na mediana. A comparação entre os portes foi feita por meio do teste de Friedman. As análises de associação entre a replicação da ENPACS e variáveis qualitativas foram feitas por meio do teste qui-quadrado de Pearson ou do teste exato de Fisher. Em todas as análises foram considerados estatisticamente diferentes testes que apresentaram probabilidades de significância inferiores a 0,05 .

A pesquisa foi aprovada pelo Comitê de Ética em Pesquisa da Universidade Federal de Minas Gerais ( ${ }^{\circ}$ CAAE 05360112.6.0000.5149). Foram respeitados todos os princípios éticos estabelecidos pelo Conselho Nacional de Saúde, contidos na Resolução $n^{\circ} 196$ de 10 de outubro de 1996. As referências técnicas que aceitaram responder ao questionário assinaram um Termo de Consentimento Livre e Esclarecido.

\section{Resultados}

\section{Sistema de Vigilância Alimentar e Nutricional Web}

Dos 39 municípios que apresentaram dado para o índice peso/idade de crianças de 0 a 23 meses no Sisvan $W e b, 6(15,4 \%)$ não apresentaram acompanhamento registrado no Sisvan Web nos anos de 2008 a 2011. O percentual de municípios que apresentaram acompanhamento registrado no Sisvan $\mathrm{Web}$ foi $61,5 \%$ em 2008, $69,2 \%$ em 2009 e $64,1 \%$ em 2010 e 2011. Em contrapartida, o percentual de municípios que apresentaram acompanhamentos provenientes do SGPBF foi $94,9 \%$ nos anos de 2008 e 2009 e $100 \%$ em 2010 e 2011.

No período de 2008 a 2011, verificou-se aumento do número de crianças de 0 a 23 meses da SRS-BH acompanhadas, anualmente, pelo Sisvan $W e b$ de acordo com o índice peso/idade. O número de crianças que tiveram acompanhamento de peso inserido no SGPBF foi maior do que o número de crianças que tiveram o acompanhamento de peso inserido no Sisvan Web nos anos de 2008 e 2010 (Tabela 1).

Observou-se variação importante no número de crianças acompanhadas, por mês, pelo índice peso/idade do Sisvan Web no período de 2009 a 2011. O total de crianças acompanhadas variou de $88(\mathrm{jan} / 2009)$ a $2268(\mathrm{mar} / 2011)$. Considerando

Tabela 1

Número de crianças de 0 a 23 meses da SRS-BH acompanhadas pelo índice peso/idade do Sisvan Web: total e por sistema de origem do registro, 2008-2011.

\begin{tabular}{lccc}
\hline Ano & $\begin{array}{c}\text { Total de crianças } \\
\text { acompanhadas } \\
\text { no Sisvan Web }\end{array}$ & $\begin{array}{c}\text { Acompanhamentos } \\
\text { inseridos diretamente } \\
\text { no Sisvan Web }\end{array}$ & $\begin{array}{c}\text { Acompanhamentos } \\
\text { provenientes do Sistema de } \\
\text { Gestão do Bolsa Família }\end{array}$ \\
\hline 2008 & 4205 & 1100 & 2392 \\
2009 & 7281 & 4487 & 2999 \\
2010 & 8326 & 3984 & 4534 \\
2011 & 8027 & 4314 & 4028
\end{tabular}

Fonte: Dados obtidos do Módulo Gerador de Relatórios do Sisvan Web, 2012; Sisvan = Sistema de Vigilância Alimentar e Nutricional. 
apenas os acompanhamentos inseridos no Sisvan Web, a variação foi 74 (jan/2009) a 1244 (abr/2009). Considerando somente os acompanhamentos provenientes do SGPBF, a variação foi 3 (jul/2009) a 1880 (mar/2011) (Figura 1).

Calculando-se as razões entre o total de crianças da SRS-BH acompanhadas, no mês, pelo índice peso/idade e o total de crianças pesadas, no mesmo mês $x 100$, foram obtidos valores que variaram entre 0,04 e 9,29 no período de 2008 a 2011.

Em relação à cobertura do Sisvan Web para crianças de 0-23 meses obtiveram-se os seguintes valores, considerando 39 municípios da regional: $4,31 \%$ em 2008; 7,54\% em 2009; $11,22 \%$ em 2010 ; e 10,69\% em 2011. Após classificação por porte, os municípios foram divididos em quatro grupos: dezessete municípios foram classificados como muito pequeno porte, nove como pequeno porte, seis como médio porte e sete como grande porte. A Tabela 2 apresenta as medianas das coberturas de acordo com o grupo/porte do município e ano. As medianas das coberturas apresentaram tendência de crescimento no tempo $(p=0,003)$. Embora o grupo de municípios classificado como de grande porte tenha apresentado medianas sistematicamente inferiores aos demais grupos, a diferença entre os grupos não se mostrou significante $(p=0,057)$.

Após subtração do número de beneficiários de planos de saúde do denominador, as coberturas do
Sisvan Web para crianças de 0-23 meses, considerando 39 municípios da regional, aumentaram para: $5,38 \%$ em 2008; 9,63\% em 2009; $16,15 \%$ em 2010 e $16,00 \%$ em 2011.

Em relação ao marcador de consumo alimentar "distribuição de crianças de 0 a 6 meses sob aleitamento materno", somente 11 municípios $(28,2 \%)$ avaliaram pelo menos uma criança no período de 2008 a 2011. A Tabela 3 apresenta o número de municípios que alimentaram o Sisvan Web e o número de crianças da SRS-BH acompanhadas por ano. Verificou-se um baixo percentual de municípios que acompanharam crianças em relação a esse marcador.

O "Questionário sobre o funcionamento do Sisvan" foi respondido por 38 referências técnicas municipais do Sisvan (20 nutricionistas, 10 enfermeiros, 5 digitadores do Sisvan, 2 assistentes sociais e 1 pediatra). Como foi enviado apenas um questionário por município, os dados consolidados são referentes a 38 municípios da SRS-BH (95\%). Desses, 33 (86,8\%) informaram que já realizaram o primeiro acesso ao Sisvan Web para inclusão de dados e $5(13,2 \%)$ informaram que nunca inseriram dados no Sisvan Web. Em relação à capacitação para implantação do Sisvan Web no município, 20 $(52,6 \%)$ informaram que foi realizada capacitação, $12(31,6 \%)$ informaram que ainda não houve capacitação e $6(15,8 \%)$ não souberam informar. Foram

Figura 1

Número de crianças de 0 a 23 meses da SRS-BH, acompanhadas pelo índice peso/idade do Sisvan Web: total e por sistema de origem do registro, Jan/2009-Dez/2011.

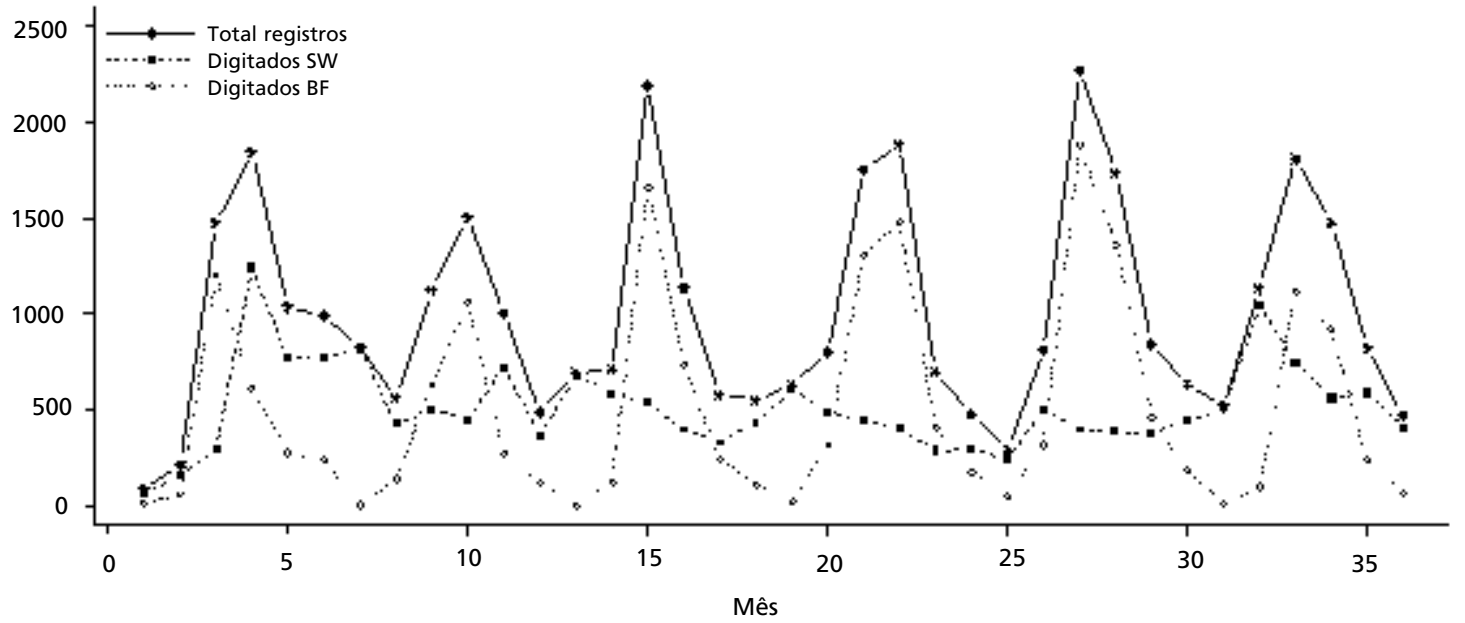

Fonte: Dados obtidos do Módulo Gerador de Relatórios do Sisvan Web, 2012; Sisvan = Sistema de Vigilância Alimentar e Nutricional. 
obtidas informações a respeito da coleta, digitação, e nutricional (Tabela 4).

análise e utilização dos dados da vigilância alimentar

.

(2)

\section{Tabela 2}

Mediana da cobertura do Sisvan Web para crianças de 0 a 23 meses, de acordo com o grupo/porte do município e ano, 2008-2011.

\begin{tabular}{llllr}
\hline \multirow{2}{*}{ Ano } & \multicolumn{3}{c}{ Porte do município } \\
\cline { 2 - 4 } & Grande & Médio & Pequeno & Muito pequeno \\
\hline 2008 & 3,16 & 3,72 & 4,04 & 7,87 \\
2009 & 5,23 & 6,70 & 8,71 & 6,63 \\
2010 & 6,62 & 11,60 & 9,59 & 17,27 \\
2011 & 8,93 & 14,27 & 15,70 & 13,61 \\
\hline
\end{tabular}

Sisvan = Sistema de Vigilância Alimentar e Nutricional.

\section{Tabela 3}

Número de municípios da SRS-BH que acompanharam criança para o marcador do Sisvan Web "distribuição de crianças de 0 a 6 meses sob aleitamento materno", 2008-2011.

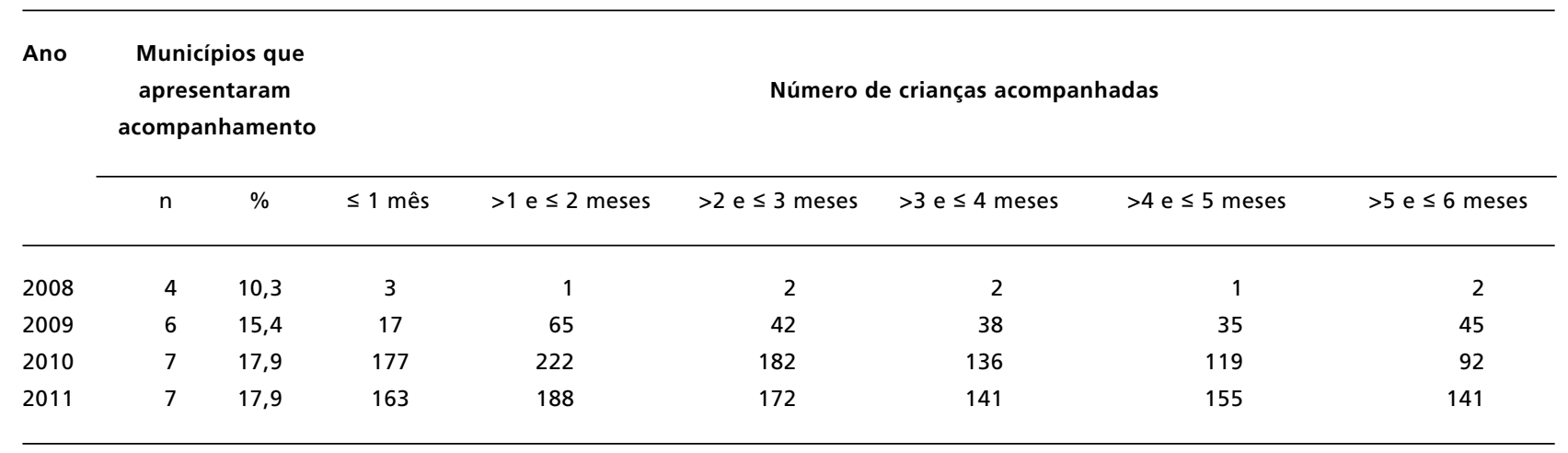

Fonte: Dados obtidos do Módulo Gerador de Relatórios do Sisvan Web, 2012; Sisvan = Sistema de Vigilância Alimentar e Nutricional. 
Informações obtidas dos questionários sobre coleta, digitação, análise e utilização dos dados da vigilância alimentar e nutricional (38 municípios da SRS-BH).

O seu município analisa os dados gerados nos relatórios do

O seu município analisa os dados gerados nos relatórios do Sisvan Web referentes ao consumo alimentar dos usuários da atenção básica?

O município estabelece ações de intervenção com base nas

\section{Estratégia Nacional para Alimentação Complementar Saudável}

Em 2010, formaram-se 49 tutores nas duas Oficinas da ENPACS promovidas pela SRS-BH, sendo 29 nutricionistas, 16 enfermeiros, 1 pediatra, 1 psicólogo, 1 assistente social e 1 técnico de enfermagem. A primeira oficina foi realizada em maio de 2010 e contou com 22 participantes. A segunda oficina foi realizada em novembro de 2010 e contou com 27 participantes. Dos 40 municípios da SRS$\mathrm{BH}, 32(80 \%)$ tiveram pelo menos um técnico formado como tutor da ENPACS.
A replicação das rodas de conversa pelos municípios iniciou-se em julho de 2010. Dos 32 municípios que tiveram pelo menos um tutor formado em Oficina da ENPACS, 16 (50\%) informaram a realização de rodas de conversa, em pelo menos uma UBS, por meio do envio da "Planilha de monitoramento da ENPACS", 4 (12,5\%) informaram que realizaram as rodas de conversa, mas não enviaram a planilha de monitoramento e $12(37,5 \%)$ informaram que não realizaram as rodas de conversa. Considerando os 16 municípios que enviaram a planilha até agosto de 2012, o percentual de UBS em que foram realizadas as rodas de conversa variou de 
$11,6 \%$ a $100 \%$, sendo que em 7 municípios $(43,7 \%)$ esse percentual foi $100 \%$. Em 4 (25\%) municípios, além das rodas de conversa, houve também a realização de oficinas para formação de novos tutores.

No que diz respeito às informações obtidas dos questionários, 17 referências $(44,7 \%)$ informaram que foram realizadas rodas de conversa em seu município. Entre os municípios em que ocorreram as rodas de conversa, $8(47,1 \%)$ informaram a realização do monitoramento da ENPACS e 9 (52,9\%) que o monitoramento não é realizado. Em relação aos instrumentos utilizados para o monitoramento da ENPACS, apenas um município informou a utilização do Sisvan Web. Os outros instrumentos utilizados foram: relatório mensal da $\mathrm{AB}$, citado por um município; Sistema on line de monitoramento da ENPACS, citado por três municípios e Planilha de monitoramento da ENPACS, citada por cinco municípios.

\section{Análises de associação}

Nas análises de associação, foi considerada a informação sobre replicação da ENPACS fornecida por 30 municípios. Entre os 14 municípios que replicaram a ENPACS, 8 (57,1\%) informaram analisar dados gerados nos relatórios do Sisvan $\mathrm{Web}$ referentes a estado nutricional e entre os 16 municípios que não replicaram, $5(31,3 \%)$ informaram analisar dados gerados nos relatórios do Sisvan Web referentes a estado nutricional, sendo a associação não significativa $(p=0,15)$. Entre os 14 municípios que replicaram a ENPACS, 7 (50\%) informaram estabelecer ações de intervenção com base nas análises dos dados do Sisvan Web e entre os 16 municípios que não replicaram, 3 (18,8\%) informaram estabelecer ações de intervenção com base nas análises dos dados do Sisvan $W e b$, sendo a associação não significativa $(p=0,12)$.

\section{Discussão}

Nos anos de 2008 a 2011, o percentual de municípios que apresentaram acompanhamentos de peso inseridos diretamente no Sisvan $W e b$ variou de $61,5 \%$ a $69,2 \%$, sendo que em $63,2 \%$ dos municípios que responderam ao questionário o Sisvan Web é acessado para digitação de dados de peso e altura. Em contrapartida, o percentual de municípios que apresentaram acompanhamentos inseridos no SGPBF variou de $94,9 \%$ a $100 \%$. Observou-se que aproximadamente $50 \%$ dos registros presentes no Sisvan $W e b$ eram provenientes do SGPBF. A priorização da alimentação do SGPBF também foi veri- ficada com a aplicação do questionário sobre o funcionamento do Sisvan. Mais de 70\% dos municípios informaram a priorização da coleta e digitação de dados de peso e altura de indivíduos beneficiários do Bolsa Família. O fato do acompanhamento semestral do estado nutricional das crianças de 0 a 23 ser uma das condicionalidades de saúde do Bolsa Família pode explicar a priorização desses dados, uma vez que as condicionalidades ${ }^{21}$ são compromissos que devem ser cumpridos para que a família possa continuar recebendo o benefício.

A priorização da alimentação de Sistemas relacionados ao recebimento de recurso também foi apontada por outros autores. Vidor et al.24 realizaram estudo sobre a utilização de SIS em municípios gaúchos de pequeno porte e verificaram que os Sistemas mais frequentemente alimentados e analisados relacionavam-se com controle orçamentário ou repasses financeiros do nível central. De acordo com Mishima et al.,25 o registro de dados nos SIS está baseado nas informações solicitadas pelos níveis centrais para fins de recebimento de recurso, sendo que ainda são raras as experiências das Secretarias de Saúde que conseguiram ampliar seus registros de acordo com as necessidades dinâmicas e específicas da população. Prevalecem registros que atendem à necessidade de financiamento, como de produção médica, procedimentos de enfermagem e vacinação.

É preconizado pelo MS que o registro do acompanhamento nutricional e alimentar das crianças de 0 a 23 meses, no Sisvan Web, siga o calendário mínimo de consultas para a assistência à criança. Logo, deve ser realizado aos 15 dias de vida, 1 mês, 2, 4, 6, 9, 12 e 18 meses. 26 No presente estudo, verificou-se que, para todos os meses, o número de crianças pesadas de acordo com o SIAB foi maior do que o número de crianças acompanhadas pelo Sisvan $W e b$ em relação ao índice peso/idade, sendo que a maior razão Sisvan/SIAB x100 foi 9,29. Com base nesse resultado, é possível inferir que muitos dados de peso coletados não são inseridos no Sisvan Web. $\mathrm{O}$ fato do SIAB ser utilizado para o registro das ações e resultados das atividades realizadas pelas Equipes de Saúde da Família pode explicar o fato de ele ter sido melhor alimentado do que o Sisvan Web.

A irregularidade no número de crianças acompanhadas, mensalmente, pelo índice peso/idade do Sisvan Web corrobora com o apontado pelos estudos de Venâncio et al. ${ }^{9}$ e Coutinho et al. 10 em relação a descontinuidade do envio de dados para o Sisvan.

Os resultados do presente estudo apontaram para as baixas coberturas do Sisvan $\mathrm{Web}$ para crianças de 0 a 23 meses nos Municípios da SRS-BH, nos anos 
de 2008 a 2011 , variando entre $4,3 \%$ a $10,7 \%$. No entanto, é importante ressaltar que houve tendência de crescimento das medianas das coberturas no período avaliado. $\mathrm{O}$ aumento do número de crianças acompanhadas e, especialmente, a redução do número de crianças na faixa etária são fatores que podem explicar o aumento das medianas. Após exclusão das crianças beneficiárias de planos de saúde do cálculo, as coberturas continuaram baixas, variando entre $5,4 \%$ a $16,0 \%$. Damé et al. 8 encontraram uma cobertura do Sisvan de $10,5 \%$ no Estado do Rio Grande do Sul, no ano de 2006, para crianças de 0 a 10 anos cobertas pela Estratégia Saúde da Família.

Verificou-se que o percentual de municípios que avaliaram crianças de 0 a 6 meses para o marcador de consumo alimentar do Sisvan Web "distribuição de crianças sob aleitamento materno", variou de $10,3 \%$ a $17,9 \%$ no período de 2008 a 2011. De acordo com os questionários, $47,4 \%$ dos municípios realizam a coleta de dados de consumo alimentar e somente $28,9 \%$ acessam o Sisvan $\mathrm{Web}$ para digitação desses dados. A partir de 2008, com a implantação do Sisvan Web, tornou-se possível o registro de dados de consumo alimentar no Sisvan. Para que dados de consumo alimentar possam ser lançados no Sisvan $W e b$ é necessário, durante a coleta, utilização de formulários próprios 27 disponíveis no site da Coordenação Geral de Alimentação e Nutrição (CGAN) do MS. O baixo percentual de municípios que alimentaram o Sistema com dados de consumo alimentar pode apontar para a necessidade de sensibilização e capacitação dos profissionais da atenção básica para a coleta desses dados e utilização dos formulários do Sisvan Web. A respeito disso, 31,6\% dos técnicos que responderam ao questionário informaram que ainda não houve capacitação para implantação do Sisvan Web em seu município. Também é importante ressaltar que, por ter sido implantado em 2008, o Sisvan Web ainda é incipiente e seus resultados são preliminares.

Em relação à análise dos dados da vigilância alimentar e nutricional, $42,1 \%$ dos municípios informaram a realização de análise dos dados gerados nos relatórios do Sisvan $W e b$ referentes a estado nutricional, sendo que, para os dados de consumo alimentar, esse percentual foi $13,2 \%$. Somente em $31,6 \%$ dos municípios que responderam ao questionário são estabelecidas ações de intervenção com base nas análises dos dados do Sisvan Web, o que corrobora com o apontado por Batista-Filho e Rissin $^{7}$ e por Castro ${ }^{12}$ em relação à pouca utilização dos dados do Sisvan. Santana e Santos 28 avaliaram o processo de implantação e execução do Sisvan em
35 municípios baianos onde o Programa "Leite é Saúde" estava funcionando. Os autores verificaram que a exigência formal de implantação do Sisvan não foi suficiente para garantir a geração e utilização local de informações sobre estado nutricional de crianças e gestantes nos municípios. O percentual de municípios que declararam analisar e utilizar os dados do Sisvan foi superior entre aqueles que replicaram a ENPACS, quando comparados com os municípios em que a replicação ainda não havia ocorrido, embora a associação não tenha sido significativa. A não obtenção de significância estatística nesta situação em particular não pode ser entendida como um desestímulo a prática da replicação, mas antes um indicativo da falta de poder do procedimento diante do pequeno número de informações disponíveis para avaliá-la.

No que diz respeito à ENPACS, três resultados encontrados nesse estudo merecem destaque: $37,5 \%$ dos municípios que tiveram tutor formado em 2010 informaram que ainda não replicaram a ENPACS; entre os municípios que replicaram a ENPACS e responderam ao questionário, $52,9 \%$ não realizam o monitoramento da Estratégia e entre os que realizam o monitoramento, apenas um município referiu à utilização do Sisvan Web. Esses achados indicam a existência de dificuldades no repasse e implantação dessa importante Estratégia de promoção da alimentação saudável na infância. Relatório técnico do Ministério da Saúde16 apontou algumas dificuldades para a implantação da ENPACS, no Brasil, tais como: falta de sensibilização e desconhecimento dos gestores de saúde e dos profissionais da $\mathrm{AB}$ sobre a importância dessa Estratégia, alta rotatividade dos profissionais da $\mathrm{AB}$ e dificuldades na utilização do Sisvan Web como instrumento de monitoramento. A não replicação da ENPACS indica que sua lógica conceitual e operativa ainda não foi internalizada pelos profissionais e gestores do SUS.

Podem-se citar algumas limitações deste estudo: a) Resultados encontrados nos 40 Municípios da SRS-BH podem não representar a realidade do Estado de Minas Gerais; b) Dados consolidados do Sisvan Web podem sofrer alterações. Como não há um prazo para que o município alimente o Sistema, com os acompanhamentos realizados, se forem coletados os dados para o mesmo índice antropométrico, faixa etária e ano em períodos diferentes, o número de indivíduos acompanhados poderá ser maior para a coleta mais recente. Isso se aplica somente aos dados inseridos diretamente no Sisvan $W e b$, visto que os dados que migraram do SGPBF permanecem os mesmos em qualquer período de coleta.

Conclusivamente, os resultados do presente estudo apontam a necessidade do fortalecimento do Sisvan, para que possa gerar informações consistentes sobre a situação alimentar e nutricional das crianças menores de dois 
anos, tornando-se, assim, adequado para o monitoramento da ENPACS. Dentre as ações para o fortalecimento do Sisvan e da ENPACS, que podem ser realizadas pelas Secretarias Municipais e Estaduais de Saúde e também pelo MS, encontram-se as capacitações e sensibilização dos gestores e profissionais do SUS a respeito da importância do Sisvan e da ENPACS.

A maioria dos municípios que participaram do presente estudo prioriza a coleta e digitação de dados antropométricos de beneficiários do Bolsa Família. Tal fato, aliado às baixas coberturas, é indício de que os dados presentes no Sisvan atendem mais a fins de recebimento de recursos do que às necessidades dinâmicas e específicas da população. Para que o Sisvan se torne um instrumento efetivo para o diagnóstico da situação alimentar e nutricional de uma população, é fundamental que contenha dados representativos desta população.

\section{Referências}

1. Brasil. Ministério da Saúde. Secretaria de Vigilância em Saúde. Guia de vigilância epidemiológica / Ministério da Saúde, Secretaria de Vigilância em Saúde. 6 ed. Brasília, DF; 2005. 816 p. (Série A. Normas e Manuais Técnicos)

2. Brasil. Conselho Nacional de Secretários de Saúde. Vigilância em Saúde / Conselho Nacional de Secretários de Saúde. Brasília, DF; 2007. 278 p. (Coleção Progestores Para entender a gestão do SUS, 6, I)

3. Brasil. Ministério da Saúde, OPAS, Fiocruz, Ministério do Desenvolvimento Social e Combate à Fome. Vigilância alimentar e nutricional - Sisvan: orientações básicas para a coleta, processamento, análise de dados e informação em serviços de saúde / [Andhressa Araújo Fagundes et al.]. Brasília: Ministério da Saúde; 2004. 120 p. (Série A. Normas e Manuais Técnicos)

4. Brasil. Ministério da Saúde. Secretaria de Atenção à Saúde. Departamento de Atenção Básica. Política Nacional de Alimentação e Nutrição / Ministério da Saúde. Secretaria de Atenção à Saúde. Departamento de Atenção Básica. Brasília, DF; 2012. 84 p. (Série B. Textos Básicos de Saúde)

5. Brasil. Ministério da Saúde. Departamento de Informática do SUS. Sisvan - Notas técnicas [Internet]. 2012 [acesso em 10 dez 2012]. Disponível em: http://tabnet.datasus.gov. $\mathrm{br} /$ cgi-win/SISVAN/CNV/notas_sisvan.html

6. Brasil. Ministério da Saúde. Secretaria de Atenção à Saúde. Departamento de Atenção Básica. Indicadores de Vigilância Alimentar e Nutricional: Brasil 2006 / Ministério da Saúde, Secretaria de Atenção à Saúde, Departamento de Atenção Básica. Brasília, DF; 2009. 142 p. (Série B. Textos Básicos de Saúde)

7. Batista-Filho M, Rissin A. Vigilância Alimentar e Nutricional: antecedentes, objetivos e modalidades. A VAN no Brasil. Cad Saúde Pública. 1993; 9 (Supl. 1): 99-105.
Para tanto, é necessário que haja maior comprometimento por parte dos gestores e profissionais do SUS em relação à alimentação desse SIS. A população também deve ser informada, por meio da mídia e divulgação pelos serviços de saúde, sobre a importância da mensuração das medidas antropométricas, da coleta de dados de consumo alimentar e do diagnóstico da situação alimentar e nutricional por meio do Sisvan. Essa medida incentivará a população a colaborar com o fornecimento de dados.

Faz-se necessário o desenvolvimento de mais estudos sobre a replicação da ENPACS e sua relação com o Sisvan. Também se faz necessário o desenvolvimento de mais estudos que possam apontar as causas das baixas coberturas e pouca utilização dos dados do Sisvan, que tem sido, para muitos municípios, mais um sistema de armazenamento de dados do que de geração de informações.

8. Damé PKV, Pedroso MRO, Marinho CL, Gonçalves VM, Duncan BB, Fisher PD, Romero ALC, Castro TG. Sistema de Vigilância Alimentar e Nutricional (Sisvan) em crianças do Rio Grande do Sul, Brasil: cobertura, estado nutricional e confiabilidade dos dados. Cad Saúde Pública. 2011; 27: 2155-65.

9. Venâncio SI, Levy RB, Saldiva SRDM, Mondini L, Stefanini MLR. Sistema de vigilância alimentar e nutricional no Estado de São Paulo, Brasil: experiência da implementação e avaliação do estado nutricional de crianças. Rev Bras Saúde Matern Infant. 2007; 7: 213-220.

10. Coutinho JG, Cardoso AJC, Toral N, Silva ACF, Ubarana JA, Aquino KKNC, Nilson EAF, Fagundes A, Vasconcellos AB. A organização da Vigilância Alimentar e Nutricional no Sistema Único de Saúde: histórico e desafios atuais. Rev Bras Epidemiol. 2009; 12: 688-99.

11. Arruda BKG. Sistema de Vigilância Alimentar e Nutricional. Frustações, desafios e perspectivas. Cad Saúde Pública. 1992; 8: 96-101.

12. Castro IRR. Vigilância Alimentar e Nutricional: limitações e interfaces com a rede de saúde. Rio de Janeiro: Editora Fiocruz; 1995.

13. Brasil. Ministério da Saúde. Informe: Uso dos formulários e registro das informações no novo Sistema Informatizado da Vigilância Alimentar e Nutricional - Sisvan Web. Brasília, DF; 2008.

14. Brasil. Ministério da Saúde. ENPACS: Estratégia Nacional Para Alimentação Complementar Saudável: Caderno Do Tutor / Ministério da Saúde, Rede Internacional em Defesa do Direito de Amamentar - IBFAN Brasil. Brasília, DF; 2010. 108 p. (Série F. Comunicação e Educação em Saúde)

15. Brasil. Ministério da Saúde. Secretaria de Atenção à Saúde. Departamento de Ações Programáticas e Estratégicas. II Pesquisa de Prevalência de Aleitamento Materno nas 
Capitais Brasileiras e Distrito Federal / Ministério da Saúde, Secretaria de Atenção à Saúde, Departamento de Ações Programáticas e Estratégicas. Brasília: Editora do Ministério da Saúde, 2009.108 p. (Série C. Projetos, Programas e Relatórios)

16. Brasil. Ministério da Saúde. Boletim ENPACS. v. 1. Brasília, DF; 2011

17. Brasil. Ministério da Saúde. Monitoramento da implementação da ENPACS: Passo a passo para facilitar a utilização do sistema de gerenciamento de implementação da Enpacs. Brasília, DF; 2011.

18. Brasil. Ministério da Saúde. Tabnet do Departamento de Informática do SUS. População Residente Minas Gerais [base de dados da Internet]. 2012 [acesso em 23 out 2012]. Disponível em: http://tabnet.datasus.gov.br/cgi/deftohtm.exe?ibge/cnv/pop mg.def.

19. Coordenação Geral de Alimentação e Nutrição do MS Módulo Gerador de Relatórios do Sisvan Web [Internet]. 2012 [acesso em: ago. 2012]. Disponível em: http://nutricao.saude.gov.br/sisvan/relatorios_publicos/

20. Brasil. Ministério da Saúde. Tabnet do Departamento de Informática do SUS. Sistema de Informação da Atenção Básica- Situação de Saúde - MG [base de dados da Internet]. 2012 [acesso em ago 2012]. Disponível em: http://tabnet.datasus.gov.br/cgi/deftohtm.exe?siab/cnv/SIA BSmg.def.

21. Brasil. Ministério da Saúde. Secretaria de Atenção à Saúde. Departamento de Atenção Básica. Manual de orientações sobre o Bolsa Família na Saúde / Ministério da Saúde, Secretaria de Atenção à Saúde, Departamento de Atenção Básica. 3 ed. Brasília, DF; 2010. 68 p. (Série A. Normas e Manuais Técnicos)
22. IBGE (Instituto Brasileiro de Geografia e Estatística). Indicadores Sociais Municipais: uma análise dos resultados do universo do Censo Demográfico 2010. Brasília, DF: Ministério do Planejamento, Orçamento e Gestão; 2011.

23. Brasil. Tabnet da Agência Nacional de Saúde Suplementar. Beneficiários de planos de saúde com até 1 ano [base de dados da Internet]. 2012 [acesso em 26 out 2012]. Disponível em: http://www.ans.gov.br/anstabnet/anstabnet/ deftohtm.exe?anstabnet/dados/TABNET_02.DEF.

24. Vidor AC, Fisher PD, Bordin R.Utilização dos sistemas de informação em saúde em municípios gaúchos de pequeno porte. Rev Saúde Publica. 2011; 45: 24-30.

25. Mishima SM, Villa TCS, Gomes ELR, Pratali MTR, Silva EM, Anselmi ML. O sistema de informações no processo gerencial dos serviços de saúde: algumas reflexões. Rev Latino-am Enf. 1996; 4: 83-9.

26. Brasil. Ministério da Saúde. Secretaria de Atenção à Saúde. Departamento de Atenção Básica. Protocolos do Sistema de Vigilância Alimentar e Nutricional - SISVAN na assistência à saúde / Ministério da Saúde, Secretaria de Atenção à Saúde. Departamento de Atenção Básica. - Brasília, DF; 2008. 61 p. (Série B. Textos Básicos de Saúde)

27. Coordenação Geral de Alimentação e Nutrição do MS Formulários de Marcadores de Consumo Alimentar [Internet]. 2012 [acesso em 12 nov 2012]. Disponível em: http://189.28.128.100/nutricao/docs/geral/marcador_consu mo_menores 5 anos.pdf

28. Santana LAA, Santos SMC. Sistema de Vigilância Alimentar e Nutricional na implementação do programa Leite é Saúde: avaliação em municípios baianos. Rev Nutr. 2004; 17: 283-90
Recebido em 8 de janeiro de 2013

Versão final apresentada em 15 de abril 2013

Aprovado em 29 de abril de 2013 